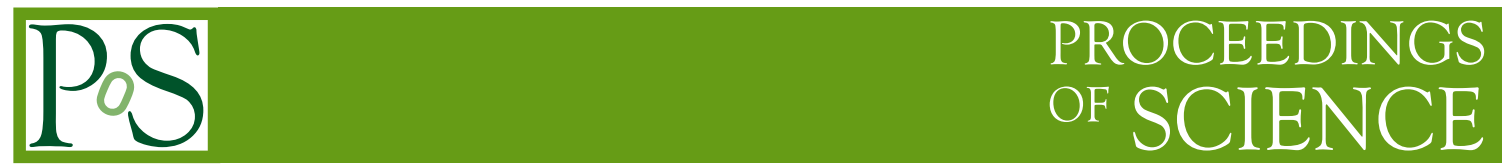

\title{
Diphoton resonance search with CMS
}

\author{
Giuseppe Fasanella* on behalf of the CMS Collaboration \\ Université Libre de Bruxelles (BE) and Sapienza Università di Roma (IT) and INFN-Roma I (IT) \\ E-mail: giuseppe.fasanella@cern.ch
}

\begin{abstract}
The results of a search for new physics using high mass diphoton events are exposed. This search employs $3.3 \mathrm{fb}^{-1}$ of pp collision data collected by the CMS experiment in 2015 at a center-ofmass energy of $13 \mathrm{TeV}$, targeting spin- 0 and spin-2 resonances of mass between 500 and 4500 $\mathrm{GeV}$ and relative width up to $5.6 \times 10^{-2}$. The results of the search are then combined with those obtained by the CMS collaboration in similar searches at $\sqrt{s}=8 \mathrm{TeV}$.
\end{abstract}

Fourth Annual Large Hadron Collider Physics

13-18 June 2016

Lund, Sweden

${ }^{*}$ Speaker. 


\section{Introduction}

The resonant production of high mass diphoton pairs is a generic prediction of several extensions of the standard model (SM) of particle physics. From very general assumptions, the spin of a resonance decaying to two photons can be restricted to be either 0 or an integer greater or equal to 2 . The production of heavy scalar resonances decaying to two photons is predicted by SM extensions with non-minimal Higgs sectors, while the production of spin-2 resonances is predicted by models postulating the existence of additional space-like dimensions. Two families of models, introduced by Arkani-Hamed, Dimopoulos and Dvali (ADD ), and by Randall and Sundrum (RS ) have been developed. In the first, the additional dimensions are assumed to be flat and compact. In the RS model, the presence of two brane-worlds is postulated and the SM fields are allowed to propagate in only one of the two. From the phenomenological point of view, both families predict that the excitations of the gravitational field lead to "towers" of spin-2 resonances, commonly denoted as gravitons, separated by a characteristic mass scale. In the case of the ADD model, the mass separation is so small that individual resonances cannot be resolved from each other. In the diphoton final state this would lead to the observation of a broad excess in the invariant mass spectrum over the SM continuum. In the case of the RS model, the mass separation is large enough to allow the independent observation of each resonance.

The results reported here are based on [1] and [2], using $3.3 \mathrm{fb}^{-1}$ of pp collisions collected by the CMS experiment in 2015 at $\sqrt{\mathrm{s}}=13 \mathrm{TeV}$.

These results are now superseded by the latest searches by ATLAS [3] and CMS [4], which are based on a factor $\approx 4$ more of integrated luminosity collected in 2016 .

\section{The CMS detector}

The central feature of the CMS apparatus is a superconducting solenoid of $6 \mathrm{~m}$ internal diameter, providing a magnetic field of 3.8 T. Within the superconducting solenoid volume area several subdetectors are installed: a silicon pixel and a strip tracker, a lead tungstate crystal electromagnetic calorimeter (ECAL), and a brass and scintillator hadron calorimeter (HCAL), each of them composed of a barrel and two endcap sections. Forward calorimeters (HF) extend the pseudorapidity coverage provided by the barrel and endcap detectors. Muons are measured in gas-ionization detectors embedded in the steel flux-return yoke outside the solenoid. A more detailed description of the CMS detector can be found in [5].

\section{Data and simulated samples}

The data considered in this analysis correspond to an integrated luminosity of $3.3 \mathrm{fb}^{-1}$ collected by the CMS experiment in 2015. Part of the dataset, corresponding to $2.7 \mathrm{fb}^{-1}$, was recorded while the CMS magnet was operated at $3.8 \mathrm{~T}$, while a second part of the dataset, amounting to $0.6 \mathrm{fb}^{-1}$, was recorded while the CMS magnet was off.

The processes giving rise to background in the search for the resonant diphoton production are the irreducible background from the direct production of two photons as well as the reducible 
components due to $\gamma+$ jets and multi-jet final states, where jet fragments are misidentified as photons. Simulated signal and background samples were used for the optimisation of the analysis and to study the systematic uncertainties related to the background estimation.

For additional details, we refer to [1].

\section{Event selection and reconstruction}

Events with at least two reconstructed photon candidates are selected and a search is made for a localised excess of events in the diphoton mass spectrum consistent with the resonant production of diphoton pairs.

Photon candidates are reconstructed from energy deposits in the ECAL. Energy deposits compatible with the expected shower shape of electrons and photons are clustered together. The clustering algorithm does not make any hypothesis as to whether the particle originating from the interaction point is a photon or an electron. Thus the same algorithm used for photon reconstruction can be applied to $\mathrm{Z} \rightarrow \mathrm{e}^{+} \mathrm{e}^{-}$events and these events can be used to measure the efficiency of the photon selection criteria and of the photon energy scale and resolution.

Photons of very high energy can deposit in a single crystals higher energy than can be measured by the ECAL digitization electronics. In this case, the estimated photon energy can be significantly lower than the true one and its variance very large. A dedicated multivariate regression was developed to correct for the effect. However, saturation occurred for none of the photon candidates selected in this analysis.

In the $\mathrm{B}=3.8 \mathrm{~T}$ dataset, the interaction vertex is selected, relying on the knowledge of the tracks momenta, while due to the lack of such information, a simpler algorithm is used for the $\mathrm{B}=0 \mathrm{~T}$ dataset and the interaction vertex with the largest track multiplicity is selected.

Photon candidates are organised in pairs, which are required to satisfy the following kinematic criteria:

- The $\mathrm{p}_{\mathrm{T}}$ of both candidates is required to be above $75 \mathrm{GeV}$.

- The absolute value of the pseudorapidity of both candidates, computed with respect to centre of the CMS detector and denoted $\left|\eta_{S C}\right|$ in the following, is required to be below 2.5 and not between 1.44 and 1.57 .

- At least one of the photon candidates is required to have $\left|\eta_{S C}\right|$ below 1.44 (i.e. events with both photon candidates in the ECAL endcaps regions are rejected).

- The invariant mass of the pair, $m_{\gamma \gamma}$, is required to be above $230 \mathrm{GeV}$. For events where one of the photon candidates is in an endcap, $m_{\gamma \gamma}>320 \mathrm{GeV}$ is required.

Photon candidates are further required to satisfy 2 specific sets of identification criteria, depending on whether the data was recorded at $\mathrm{B}=3.8 \mathrm{~T}$ or at $\mathrm{B}=0 \mathrm{~T}$. For the $\mathrm{B}=0 \mathrm{~T}$ dataset, also the transverse size of the electromagnetic cluster in the $\phi$ direction can be used as signal vs background discriminating variable. In the $\mathrm{B}=3.8 \mathrm{~T}$ dataset the efficiency of the identification criteria for prompt isolated photon candidates, in the kinematic range considered by the analysis, is above 90 (85)\% in the barrel (endcaps). In the B $=0 \mathrm{~T}$ dataset, the identification efficiency is above about 
85 (70)\% for prompt isolated photon candidates in the barrel (endcaps). Diphoton pairs are split into two categories: the first, denoted "EBEB" in the following, contains pairs where both candidates are reconstructed in the ECAL barrel, while the second, denoted "EBEE", contains pairs where one of the candidates is reconstructed in an ECAL endcap. The fraction of signal events selected in each of the analysis categories is shown in Fig. 1 for different signal hypotheses.
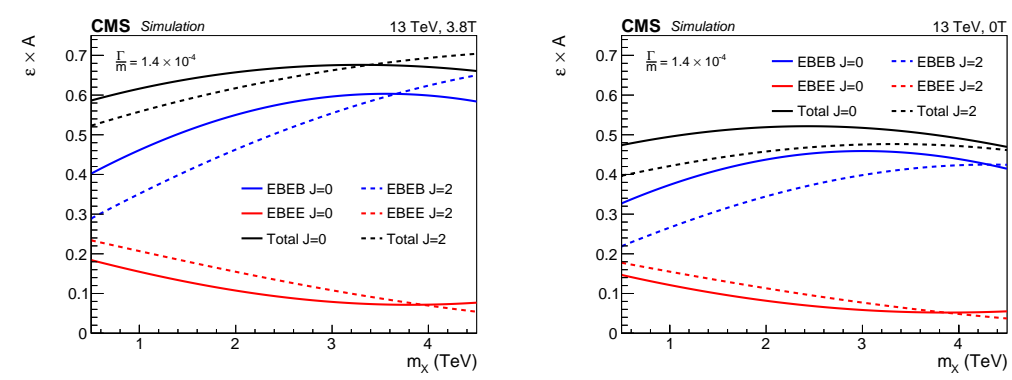

Figure 1: Fraction of events selected by the analysis categories for $500 \mathrm{GeV}<m<4.5 \mathrm{TeV}$ and $\Gamma / m=$ $1.4 \times 10^{-4}$. Curves for both spin- 0 and RS graviton resonances are shown, on the left for the $\mathrm{B}=3.8 \mathrm{~T}$ sample and on the right for the $\mathrm{B}=0 \mathrm{~T}$ one.

\section{Determination of the photon energy scale and resolution}

Discrepancies in the photon energy scale and resolution between data and simulation are resolved using dielectron events. Energy scale and resolution corrections are derived primarily from $\mathrm{Z} \rightarrow \mathrm{e}^{+} \mathrm{e}^{-}$events. The corrections are derived in eight bins defined in terms of the $R_{9}$ variable (defined as the ratio between the energy deposited in the central $3 \times 3$ crystal matrix and the full cluster energy) and of $\left|\eta_{S C}\right|$.

The size of the energy scale corrections derived from $\mathrm{Z} \rightarrow \mathrm{e}^{+} \mathrm{e}^{-}$events is of the order of $0.5(1.5) \%$ for photon candidates in the $\mathrm{B}=3.8 \mathrm{~T}(\mathrm{~B}=0 \mathrm{~T})$ dataset, while the additional Gaussian smearing needed to match the energy resolution in simulated events with that in data varies between roughly $0.8 \%$ and $1.5 \%$ for photon candidates in the ECAL barrel region and between $2 \%$ and $2.5 \%$ for photon candidates in the endcap regions. The dielectron invariant mass distribution obtained, for data and simulated events, after the adjustment procedure is shown in Fig. 2.

The stability of the correction factors as a function of the energy of the photon candidate is assessed using $\mathrm{Z} \rightarrow \mathrm{e}^{+} \mathrm{e}^{-}$events in which the $\mathrm{Z}$ boson is produced at high transverse momentum. This allows to test the transverse energy range up to roughly 150 (100) GeV in the barrel (endcap) region. For the barrel region in the both the $\mathrm{B}=3.8 \mathrm{~T}$ and $\mathrm{B}=0 \mathrm{~T}$ datasets, the energy scale corrections are found to be stable within $0.5 \%$ in the probed range. A stability better than $0.7 \%$ is observed for the photon candidates in the endcaps.

\section{Diphoton mass spectrum}

The invariant mass distribution of the selected events is shown in Fig. 3. A parametrisation of the spectrum of the form $f\left(m_{\gamma \gamma}\right)=m_{\gamma \gamma}^{a+b \cdot \log \left(m_{\gamma \gamma}\right)}$, obtained through an unbinned maximum likelihood fit to the selected events, is shown. This parametric form corresponds to the one chosen to model the background in the hypothesis tests, as detailed in Section 9. 

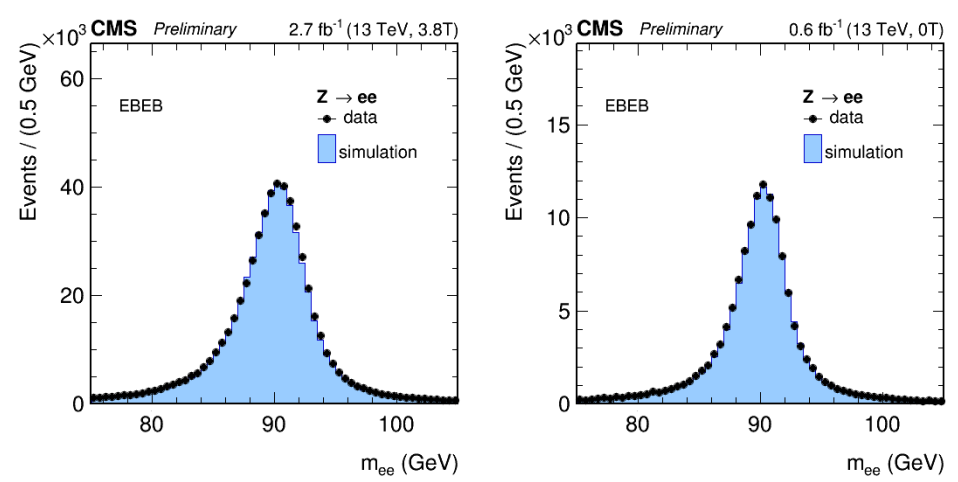

Figure 2: Comparison between the predicted and observed invariant mass distribution of electron pairs obtained after the application of energy scale and resolution corrections. Pairs of photon candidates satisfying the analysis identification criteria and compatible with electrons tracks are selected. Distributions are shown for events where both electrons are reconstructed in the barrel. The left (right) one refers to the $\mathrm{B}=3.8 \mathrm{~T}$ $(\mathrm{B}=0 \mathrm{~T})$ datasets.

\section{Statistical analysis}

The results of the search are interpreted in the frame of a composite statistical hypothesis test. A simultaneous fit to the invariant mass spectra of the EBEB and EBEE event categories is used to study the compatibility of the data with the background-only and the signal+background hypotheses.

The test statistics used in the hypothesis tests are based on the profile likelihood ratio:

$$
q(\mu)=-2 \log \frac{L\left(\mu \cdot S+B \mid \underline{\hat{\theta}}_{\mu}\right)}{L(\hat{\mu} \cdot S+B \mid \underline{\hat{\theta}})}
$$

where $S$ and $B$ are the probability density functions for the resonant diphoton production process and the SM backgrounds respectively, $\mu$ is the so-called "signal strength" parameter and $\underline{\theta}$ are the nuisance parameters of the model, used to model systematic uncertainties. The $\hat{x}$ notation indicates the best-fit value of the parameter $\mathrm{x}$, while the notation $\hat{x}_{y}$ denotes the best-fit value of $\mathrm{x}$ conditional on $\mathrm{y}$.

\section{Signal modelling}

The signal distribution in $m_{\gamma \gamma}$ is determined from the convolution of the intrinsic shape of the resonance and the ECAL detector response. The intrinsic shape of the resonant signals are derived using the PYTHIA generator. A fine grid of mass points with $125 \mathrm{GeV}$ spacing is used and the resulting shapes interpolated to the intermediate points. The detector response is determined using fully simulated signal samples of small intrinsic width and corrected for the additional Gaussian smearing determined from dielectron events. In order to determine the signal normalisation, the efficiency of the final event selection is combined with the kinematic acceptance. 

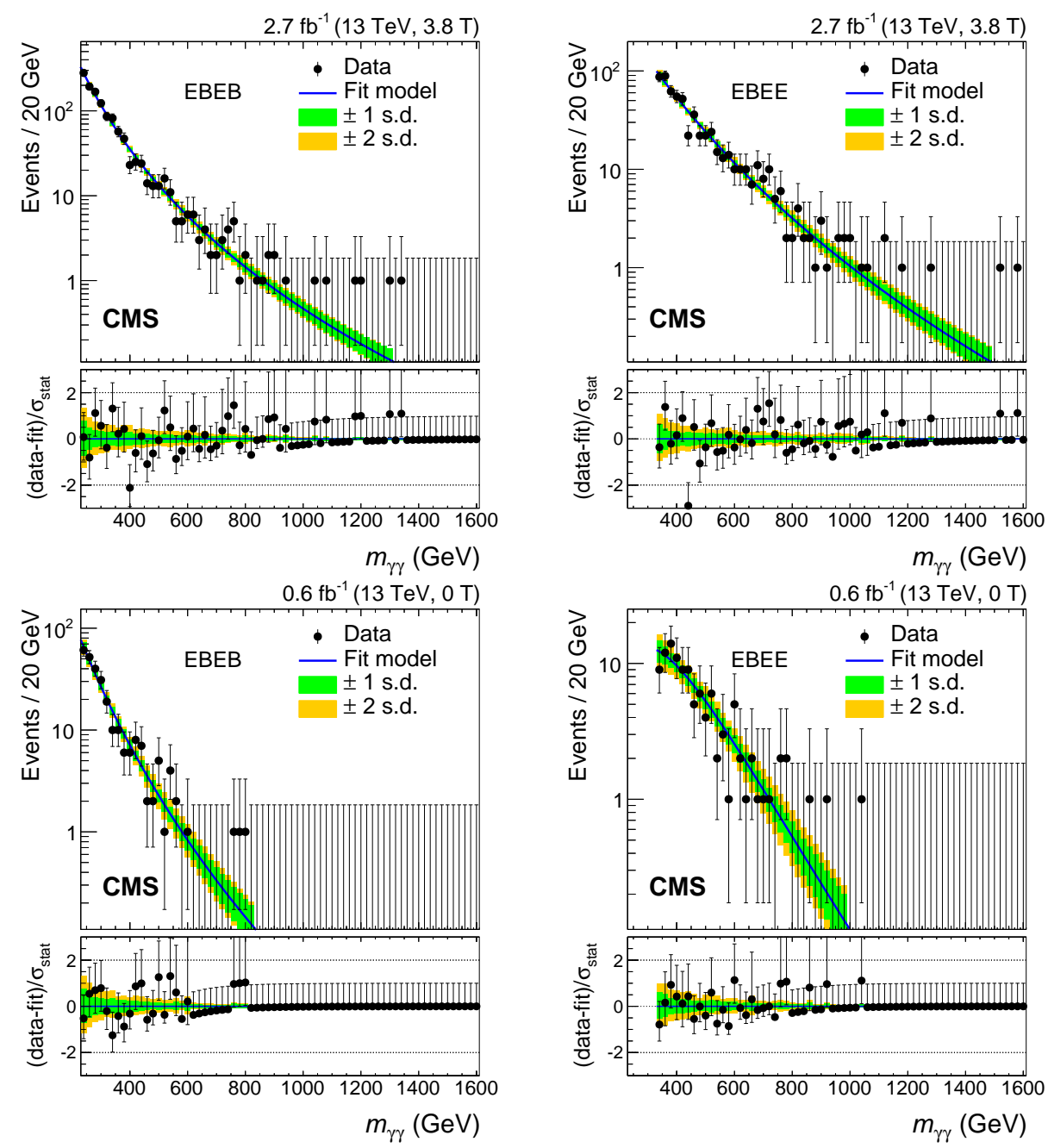

Figure 3: Observed invariant mass spectra for the EBEB (left) and EBEE (right). Top (bottom) row shows the $\mathrm{B}=3.8 \mathrm{~T}(\mathrm{~B}=0 \mathrm{~T})$ dataset. The results of parametric fits to the data are also shown.

\section{Background modelling}

The background $m_{\gamma \gamma}$ spectrum is described by a parametric function of $m_{\gamma \gamma}$. The parametric coefficients are obtained from a fit to the data events, and considered as unconstrained nuisance parameters in the hypothesis test, allowing the building a data-driven description of the shape.

The accuracy of the background determination is assessed using MC simulations and it is quantified by studying the difference between the true and predicted number of background events in several $m_{\gamma \gamma}$ windows in the search region, via pseudo-experiments.

\section{Systematic uncertainties}

In this analysis the impact of the systematic uncertainties is smaller than that of the statistical uncertainties. The shape coefficients are treated as unconstrained nuisance parameters, thus the associated uncertainties contribute to the statistical uncertainty. 
Uncertainties associated to the signal modelling are summarised here:

- Luminosity uncertainty. For the $\mathrm{B}=3.8 \mathrm{~T}$ dataset, a $2.7 \%$ on the signal normalisation was assigned, for the $\mathrm{B}=0 \mathrm{~T}$ dataset a $12 \%$ uncertainty was used instead.

- Selection efficiency uncertainties. For $B=3.8 \mathrm{~T}$, an $8 \%$ uncertainty was used, while for the $\mathrm{B}=0 \mathrm{~T}$ dataset a $16 \%$ uncertainty was used.

- Parton distribution functions. A $6 \%$ uncertainty on the signal normalisation was assigned.

- Photon energy scale uncertainty. For both the $\mathrm{B}=3.8 \mathrm{~T}$ and $\mathrm{B}=0 \mathrm{~T}$ dataset a $1 \%$ energy scale uncertainty was assign.

- Photon resolution uncertainty. The uncertainty on the resolution corrections factors is evaluated summing and subtracting $0.5 \%$ in quadrature from the estimated additional Gaussian smearing.

\section{Results of the search at $13 \mathrm{TeV}$}

To set upper limits on the resonant diphoton production rate, the modified frequentist method, commonly known as $\mathrm{CL}_{\mathrm{s}}$, has been employed using asymptotic formulae. Expected and observed upper limits on the production of scalar and RS graviton resonances are shown in Fig. 4 for values $\Gamma / m$ of $1.4 \times 10^{-4}, 1.4 \times 10^{-2}$ and $5.6 \times 10^{-2}$.
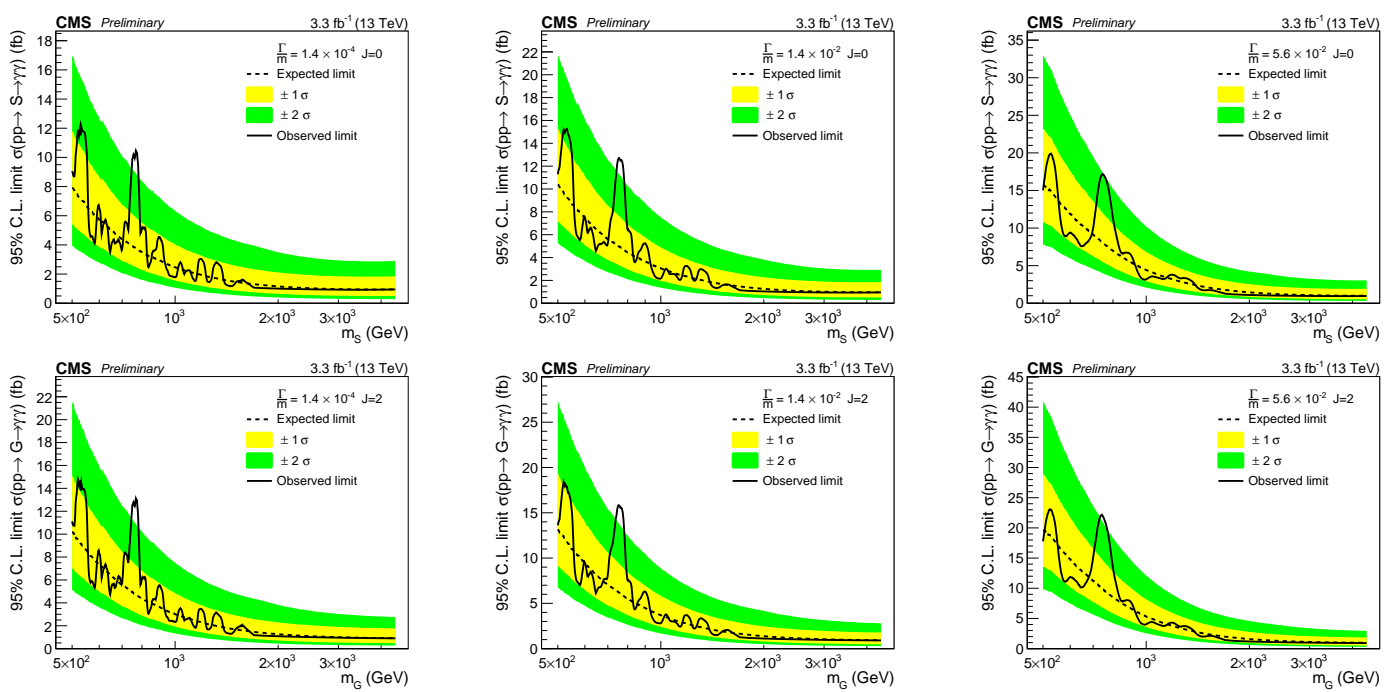

Figure 4: Expected and observed 95\% C.L. exclusion limits for different signal hypotheses. The range $500 \mathrm{GeV}<m<4.5 \mathrm{TeV}$ is shown for $\Gamma / m=1.4 \times 10^{-4}, 1.4 \times 10^{-2}, 5.6 \times 10^{-2}$. The top (bottom) row corresponds to the scalar (RS graviton) signals.

The compatibility of the observation with the background-only hypothesis is evaluated computing the background-only $p$-value. The latter is defined as the probability, in the background-only hypothesis, for $q(0)$ to be above the observation. 
The value of $p_{0}$ for different signal hypotheses is shown in Fig. 5. The largest excess observed in data has a $p_{0}$ value corresponding to 2.9 standard deviations is observed when testing for the production of an RS graviton with $m_{G}=760 \mathrm{GeV}$ and $\Gamma / m=1.4 \times 10^{-2}$.
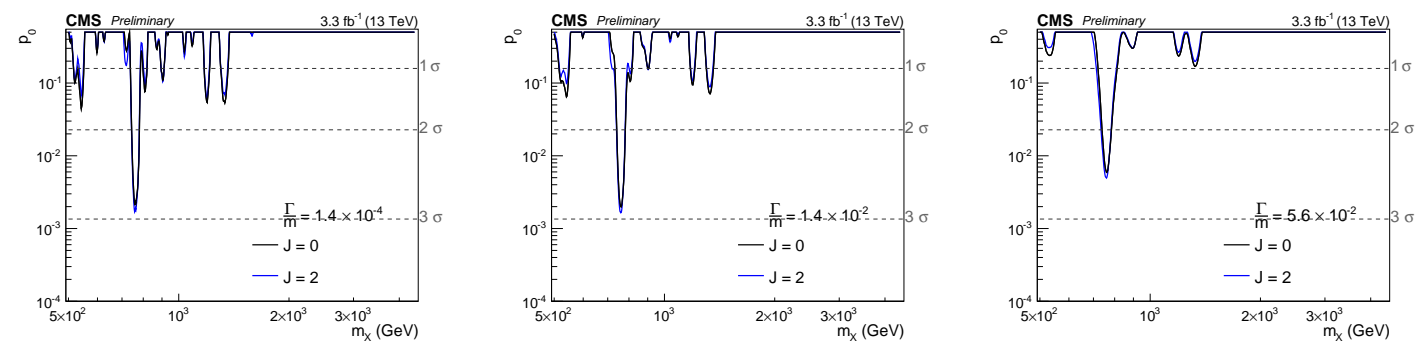

Figure 5: Observed background-only $p$-value for different signal hypotheses. The range $500 \mathrm{GeV}<m<4.5$ $\mathrm{TeV}$ is shown for $\Gamma / m=1.4 \times 10^{-4}, 1.4 \times 10^{-2}, 5.6 \times 10^{-2}$. Results corresponding to both the scalar and RS graviton hypotheses are shown.

Figure 6 shows, for $\Gamma / m=1.4 \times 10^{-2}$, the contributions of the $\mathrm{B}=3.8 \mathrm{~T}$ and $\mathrm{B}=0 \mathrm{~T}$ dataset to the observed result in the mass range up to $850 \mathrm{GeV}$.
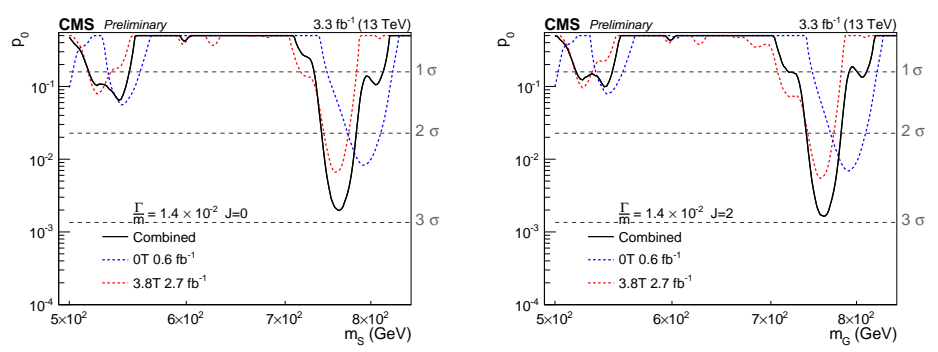

Figure 6: Observed background only p-values obtained on the $13 \mathrm{TeV}$ dataset. The mass range $500 \mathrm{GeV}$ $<m<850 \mathrm{GeV}$ is shown is for resonances of $\Gamma / m=1.4 \times 10^{-2}$. The contributions of the $\mathrm{B}=3.8 \mathrm{~T}$ and $\mathrm{B}=0 \mathrm{~T}$ datasets are shown separately. Due to the different size of the two datasets, the weight of the $\mathrm{B}=0 \mathrm{~T}$ categories in the combined result is, at $m=760 \mathrm{GeV}$, roughly one fifth of that of the $\mathrm{B}=3.8 \mathrm{~T}$ ones. The left (right) plot corresponds to the scalar (RS graviton) hypotheses.

The "local $p$-value" $p_{0}$ does not take into account the fact that many signal hypotheses are tested. The probability of observing an excess more significant than this for at least one of the signal spin and width hypotheses in the mass range between $500 \mathrm{GeV}$ and $4.5 \mathrm{TeV}$ is then estimated constructing the sampling distribution of $\max \left(p_{0}\right)$ on an ensemble of background-only pseudoexperiments. The significance of the excess is estimated to be less than one standard deviation after taking this into account.

\section{Combined analysis of $\sqrt{\mathrm{s}}=\mathbf{8} \mathrm{TeV}$ and $\mathbf{1 3} \mathrm{TeV}$ datasets}

The results obtained on the $13 \mathrm{TeV}$ dataset are combined statistically with those obtained on the data recorded by the CMS experiment at the centre of mass energy of $8 \mathrm{TeV}$. Two analyses were performed by the CMS collaboration to search for diphoton resonances using the $8 \mathrm{TeV}$ dataset. The analysis described in [6] searched for diphoton resonances in the mass range between 
150 and $850 \mathrm{GeV}$, interpreting the results both in terms of production of scalar particles and in terms of production of RS gravitons. The analysis described in [7] focused on the mass range above $500 \mathrm{GeV}$ and on the RS graviton hypothesis. The statistical combination of the results is performed for all signal hypotheses which are tested for the $13 \mathrm{TeV}$ analysis. The ratio of the signal production cross sections at $\sqrt{\mathrm{s}}=8 \mathrm{TeV}$ and $13 \mathrm{TeV}$ has been calculated using PYTHIA . It is roughly 0.29 (0.27) for $m_{G}\left(m_{S}\right)=500 \mathrm{GeV}$ and decreases to $0.04(0.03)$ when $m_{G}\left(m_{S}\right)=3 \mathrm{TeV}$. Since the event samples selected by the two $8 \mathrm{TeV}$ analyses partially overlap, only one of the two (the one giving the most stringent median expected exclusion limit) is considered for each hypothesis test.

The expected and observed median 95\% C.L. exclusion limits on the equivalent $13 \mathrm{TeV}$ production cross section, $\sigma_{G, S}^{13 \mathrm{TeV}} \cdot \mathscr{B}_{\gamma \gamma}$ for the combined analysis are shown in Figure 7. For the signal hypotheses below roughly $1.5 \mathrm{TeV}$, the exclusion limits obtained with the combined analysis are more stringent than those obtained individually on the 8 or $13 \mathrm{TeV}$ datasets by $20-40 \%$. In the region above $1.5 \mathrm{TeV}$ the exclusion limit is determined mostly by the $13 \mathrm{TeV}$ analysis.

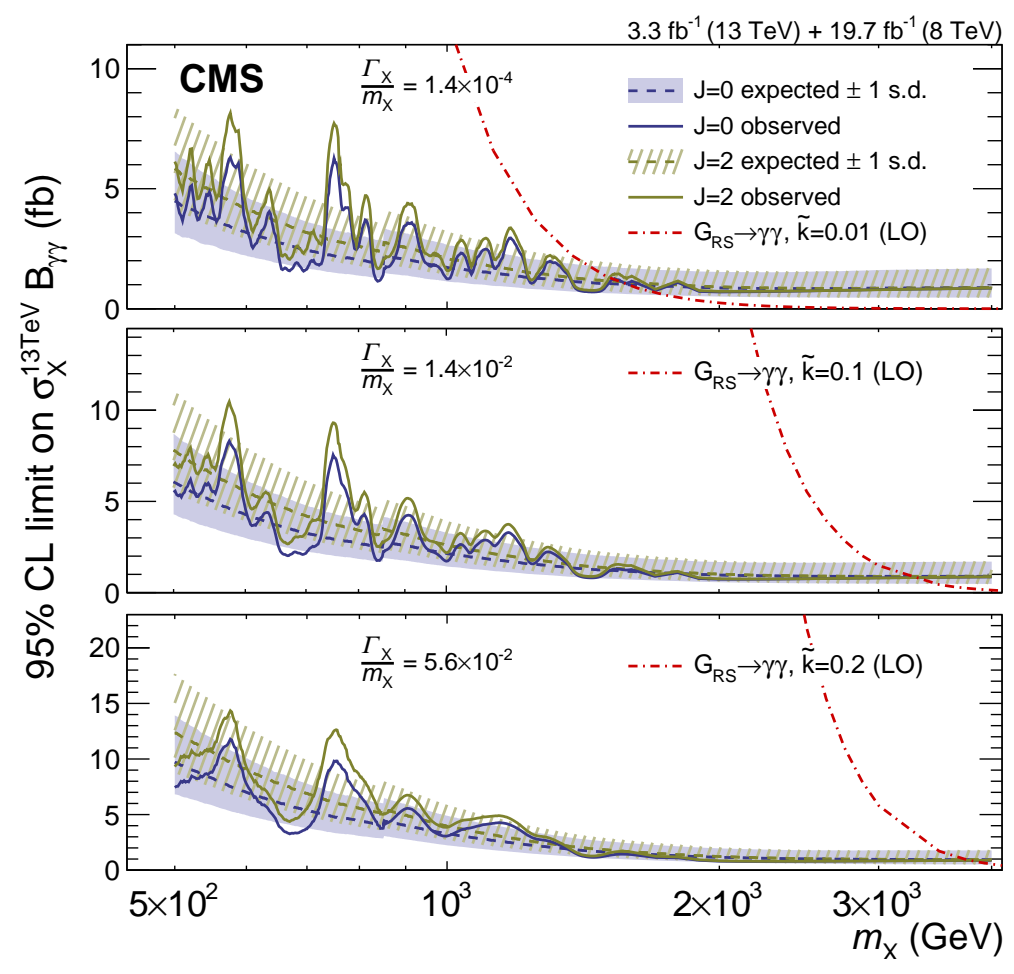

Figure 7: The 95\% CL upper limits on the production of diphoton resonances as a function of the resonance mass $m$, from the combined analysis of the 8 and $13 \mathrm{TeV}$ data. The $8 \mathrm{TeV}$ results are scaled by the ratio of the 8 to $13 \mathrm{TeV}$ cross sections. The blue-grey (darker) curves and the green (lighter) ones correspond to the scalar and RS graviton signals, respectively. Solid (dashed) curves represent the observed (median expected) exclusion limit. The expected results are shown with their 1 standard deviation dispersion bands. The leading-order RS graviton production cross section is shown by the red dot-dashed curves. The results are shown for (upper) a narrow, (middle) an intermediate-width, and (lower) a broad resonance, with the value of the width $\Gamma / m$, relative to the mass, indicated in the legend of each plot.

The background only $p$-value, $p_{0}$, for the combined analysis is shown in Figure 8, for all the 
tested signal hypotheses. The largest excess is observed for the narrow width hypothesis at $m_{S}=$ $750 \mathrm{GeV}$ and has a local significance of approximately 3.4 standard deviations, which reduce to approximately 1.6 standard deviations, when taking into account the effect of testing all the signal hypotheses considered.
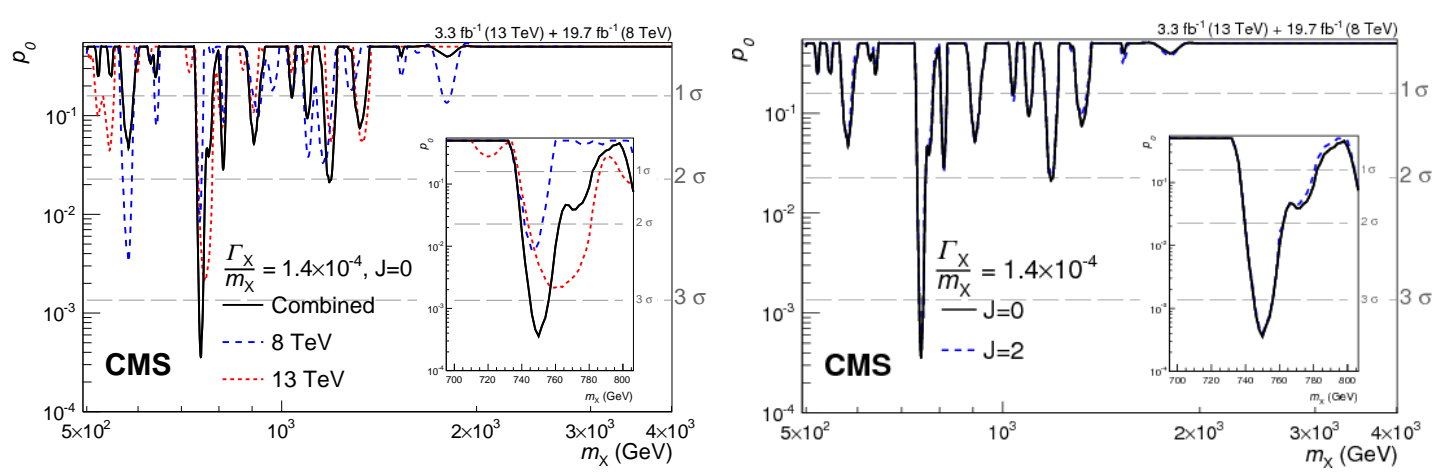

Figure 8: Observed background only p-values obtained from the combination of $8 \mathrm{TeV}$ and $13 \mathrm{TeV}$ results for different signal hypotheses. The contributions of the 8 and $13 \mathrm{TeV}$ datasets are shown separately in the left plot for the scalar resonance scenario $(\mathrm{J}=0)$, while in the right plot the comparison between the $\mathrm{p}$-values obtained in the scenarios $\mathrm{J}=0$ and $\mathrm{J}=2$ is shown .

To further qualify the compatibility of the results obtained with $8 \mathrm{TeV}$ and $13 \mathrm{TeV}$ datasets, we compute the likelihoods of the fits to a signal plus background hypothesis as a function of the equivalent cross section (extrapolated to $\sqrt{\mathrm{s}}=13 \mathrm{TeV}$ ). Narrow scalar and RS graviton hypotheses with mass $m=750 \mathrm{GeV}$ were chosen. The fits are performed separately for each of two datasets and for the combined analysis. The results are shown in Fig. 9, where it can be seen that the equivalent cross sections estimated for the two datasets are compatible with each other, under both spin hypotheses.
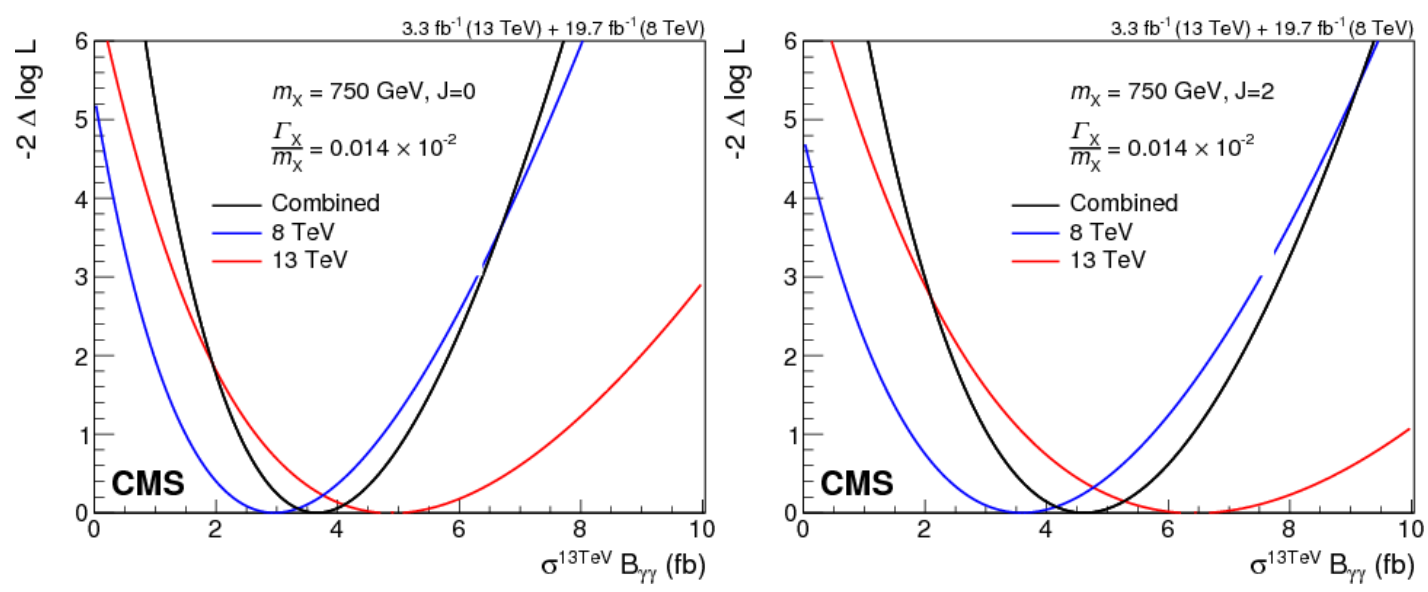

Figure 9: Likelihood scan for the cross section corresponding to the largest excess in the combined analysis of the 8 and $13 \mathrm{TeV}$ datasets. The left (right) column corresponds to the scalar (RS graviton) signals. The 8 $\mathrm{TeV}$ results are scaled by the expected ratio of cross sections in each scenarios. 


\section{Summary}

A search for new physics using the diphoton mass spectrum has been presented. The analysis is based on $3.3 \mathrm{fb}^{-1}$ of pp collisions collected by the CMS experiment in 2015 at $\sqrt{\mathrm{s}}=13 \mathrm{TeV}$. Events containing two photon candidates with transverse momenta above $75 \mathrm{GeV}$ were selected. The mass spectrum above $500 \mathrm{GeV}$ was inspected to search for the production of spin- 0 and spin- 2 resonances.

Limits on the production of scalar resonances and Randall-Sundrum gravitons in the range where $500 \mathrm{GeV}<m<4.5 \mathrm{TeV}$ and $\Gamma / m<5.6 \times 10^{-2}$ were set using the modified frequentist approach. The largest excess is observed for $m=760 \mathrm{GeV}$ and $\Gamma / m=1.4 \times 10^{-2}$ and has a local significance of 2.8-2.9 standard deviations depending on the spin hypothesis. After taking into account the effect of searching for several signal hypotheses, the significance of the excess is reduced to less than one standard deviation.

The results of the search are combined statistically with those obtained in similar searches at $\sqrt{\mathrm{s}}=8 \mathrm{TeV}$ in the mass range $500 \mathrm{GeV}<m<3.5 \mathrm{TeV}$ and $\Gamma / m<5.6 \times 10^{-2}$. The combined analysis improves the sensitivity of the individual analyses by $20-40 \%$ in the mass range up to roughly $2.5 \mathrm{TeV}$. The largest excess is observed in the combined analysis for $m=750 \mathrm{GeV}$ and $\Gamma / m=1.4 \times 10^{-4}$. The local $p$-value corresponds to approximately 3.4 standard deviations, which are reduced to approximately 1.6 standard deviations after taking into account the effect of searching for several signal hypotheses.

\section{References}

[1] The CMS Collaboration, Search for new physics in high mass diphoton events in $3.3 \mathrm{fb}^{-1}$ of proton-proton collisions at $\sqrt{(s)}=13 \mathrm{TeV}$ and combined interpretation of searches at $8 \mathrm{TeV}$ and 13 $\mathrm{Te} V$, CMS-PAS-EXO-16-018

[2] The CMS Collaboration, Search for resonant production of high-mass photon pairs in proton-proton collisions at $\sqrt{(s)}=8$ and 13 TeV, Phys.Rev.Lett. 117 (2016) no.5 [arXiv: 1606.04093 ]

[3] The ATLAS Collaboration, Search for scalar diphoton resonances with $15.4 \mathrm{fb}^{-1}$ of data collected at $\sqrt{(s)}=13 \mathrm{TeV}$ in 2015 and 2016 with the ATLAS detector, ATLAS-CONF-2016-059

[4] The CMS Collaboration, Search for resonant production of high mass photon pairs using $12.9 \mathrm{fb}^{-1}$ of proton-proton collisions at $\sqrt{s}=13 \mathrm{TeV}$ and combined interpretation of searches at 8 and $13 \mathrm{TeV}$, CMS-PAS-EXO-16-027

[5] CMS Collaboration, The CMS experiment at the CERN LHC, JINST 3 (2008) S08004, 558

[6] CMS Collaboration, Search for diphoton resonances in the mass range from 150 to $850 \mathrm{GeV}$ in pp collisions at $\sqrt{(s)}=8$ TeV, Phys. Lett. B 750 (2015) 494-519 [arXiv: 1506.02301 ]

[7] CMS Collaboration, Search for High-Mass Diphoton Resonances in pp Collisions at $\sqrt{(s)}=8 \mathrm{TeV}$ with the CMS Detector, CMS-PAS-EXO-12-045 